\title{
The unseen story of tinidazole: alarm to the drug regulatory bodies
}

\begin{abstract}
Tinidazole is a drug of choice for many aerobic or anaerobic bacterial infections. But, its adverse reactions affect almost all body systems. Several studies of tinidazole were reviewed which suggests that use of tinidazole leads to hypersensitivity or anaphylactic reactions, fixed drug eruptions, rash, erythema, Stevens Johnson Syndrome etc. Again, the rationality of fixed dose combination of tinidazole is controversial. It is a widely debated issue in clinical practice of India. So, alertness of both, the patient and the physician is important towards promoting safe and rational use of tinidazole. Also, drug regulatory bodies should take urgent action to mitigate the rampant use of irrational fixed dose combinations of tinidazole.
\end{abstract}

Keywords: tinidazole, fixed drug eruptions, stevens johnson syndrome, irrational fixed dose combinations
Volume 2 Issue I - 2015

\author{
Himanshu Bhusan \\ MKCG Medical College \& Hospital, India
}

Correspondence: Himanshu Bhusan, MKCG medical College \& hospital, Orissa, India, Tel 9439893903,

Email bhusan.himanshu@yahoo.co.in

Received: January 12, 2015 | Published: January 27, 2015

\section{Introduction}

Tinidazole (1-[2-(ethylsulfonyl)ethyl]-2-methyl-5-nitroimidazole) was first introduced into clinical medicine in 1967 and has been used in Europe, Australia, Asia and in a number of developing countries for decades. It was approved by the FDA for the treatment of protozoal infections like trichomoniasis, giardiasis, intestinal amoebiasis, amoebic liver abscess. It is also very effective for bacterial vaginosis, endometritis, dental infections, peptic ulcer by $H$. pylori and chemoprophylaxis in gynaecological, colorectal, oral and maxio-facial surgery. ${ }^{1}$ It is used as the drug of choice for various infectious diseases because of its better efficacy, desirable tolerance, an option for single dose regimen, short course of therapy and good patient compliance. In spite of being increasingly used in different dosage regimens for various protozoal and bacterial infections for the last 25 years, mild to moderate adverse reactions occur when it is used alone as well as in fixed dose combinations. This has been observed across various dose ranges, routes of administration and age groups. Severe and potential life-threatening reactions like urticaria, laryngo-facial oedema, hypotension, bronchospasm, dyspnoea and anaphylactic reactions due to tinidazole have been widely reported in scientific literature. ${ }^{2}$ The development of mild allergic reaction at the initial dose of tinidazole and severe anaphylaxis on re-exposure even after eighteen months have been reported. In previously exposed patients, reactions on re-exposure are rapid and severe. Activation of mast cells and basophils by allergen specific $\operatorname{IgE}$ is the basis for such reactions. The ethyl-sulfonyl group of tinidazole is primarily responsible for the hypersensitivity and anaphylactic reactions.

\section{Adverse events associated with tinidazole used in clinical practice}

Various case reports were reported regarding tinidazole, when used alone or as fixed dose combination or in various dose ranges induces mild to moderate adverse reactions to every age groups. ${ }^{2}$ In a case study, generalized itching with a solitary lesion on the genitals was observed on the $2^{\text {nd }}$ day after administration of $1000 \mathrm{mg}$ once daily per orally. The case was diagnosed as fixed drug eruption considering the temporal relation with the drug consumption. ${ }^{3}$ Another study reported that the patient developed generalized itching and ulcerations on the lips and trunk on the $4^{\text {th }}$ day after taking $400 \mathrm{mg}$, twice daily per oral for dysentery. ${ }^{4}$ In one study, with the administration of $500 \mathrm{mg}$ twice daily per oral for the treatment of amoebiasis, hyperpigmentation of both the lips was observed on the $2^{\text {nd }}$ day. ${ }^{5}$ Developments of multiple voliaceous, erythematous, itchy, well demarcated lesions on the dorsa of the hands and penis after six hours of intake of $300 \mathrm{mg}$ have been reported. In this case study after the primary lesions subsided, rechallenge with $300 \mathrm{mg}$ per oral was done and it was found that within five hours, there was burning and tingling sensation in the previously affected areas. ${ }^{6}$ In another case report, the dusky rounded patches, surrounded by erythema developed within four hours of administration of tinidazole per oral. After two months, the same patient developed fixed drug eruptions on his hands, lips and glans penis after per oral administration of tinidazole for dysentery. In both the instances the lesions cleared spontaneously without pigmentation after three weeks. ${ }^{6}$ In a case report, the patient developed severe anaphylaxis characterised by severe bronchospasm, laryngeal oedema, tachycardia, hypotension and difficulty in talking and swallowing within an hour of ingestion of $250 \mathrm{mg}$ (half tablet of tinidazole $500 \mathrm{mg}$ ). Further administration of the drug was stopped. On the next day, severe oral and genital mucosal lesions were seen. On hospitalisation and it was diagnosed as a case of tinidazole induced anaphylaxis and Steven-Johnson syndrome. ${ }^{2}$ In a pharmacokinetic study of tinidazole, the clinical volunteer fainted and lost consciousness for 10 seconds on administration of tinidazole $1600 \mathrm{mg}$ IV infusion. The attack was followed by hypotension, nausea and fatigue. It was concluded that tinidazole caused an acute toxic reaction with subsequent activation of complement factors. ${ }^{7}$

Tinidazole is also marketed as a fixed dose combination with norfloxacin, dicyclomine, loperamide, ofloxacin, ciprofloxacin, doxycycline, fluconazole etc. ${ }^{8}$ In a study, a female patient developed itchy, erythematous, edematous, hyperpigmented plaques over the face and body on the $2^{\text {nd }}$ day of oral administration of a fixed drug combination of ciprofloxacin 500mg and tinidazole 500mg. ${ }^{4}$ In another case study, the patient developed anaphylactic reaction characterised by tachycardia, bronchospasm and laryngeal oedema within four hours of ingestion of a fixed dose combination of norfloxacin 400mg and tinidazole $500 \mathrm{mg}$ tablet. On the $2^{\text {nd }}$ day, oral mucosal lesions and 
generalised erythematous lesions developed. It was diagnosed as drug-induced anaphylaxis and erythema multiforme. ${ }^{2}$

\section{Discussion}

In all the case studies cited re-challenge was not done, probably fearing serious complications and ethical constrains. These adverse drug reactions to tinidazole can be labelled as 'Probable/likely' by causality assessment. These were not dose-dependent. They could be labelled as Type-B class of adverse drug reactions. In all the cases there was a temporal association. Fixed drug combinations of tinidazole are not recommended either by any standard text books, reference books or by any reputed medical journals. Irrational fixed dose combinations may increase chances of adverse drug reactions due to drug interactions and also lead to the emergence of antimicrobial resistance. Moreover, they also impose an unnecessary financial burden on the patients. In case, adverse reactions occur due the use of such fixed drug combinations the prescriber could be subject to litigation, in the absence of any scientific recommendation for such combinations. ${ }^{2}$ Diagnostic imprecision, ignorance of the microbial sensitivity pattern and the lack of access to laboratory facilities may be the cause of increasing use of fixed drug combinations. Fixed drug combinations of tinidazole are heavily prescribed in GI infections, pelvic inflammatory disease, dental infections. Elaborate scientific studies are required to establish the rationale of such fixed dose combinations. Pharmaceutical manufacturers, however, continue to promote such combinations with vigour to reap the benefits of huge sales. Drug regulatory authorities should be proactive to check the promotion of irrational fixed dose combinations of tinidazole.

\section{Conclusion}

Further, keeping in view the frequency of occurrence of adverse drug reactions, it is for the prescribers to be cautious in using tinidazole. Patients allergic to one azole drug should not be advised another azole drug, irrespective of the indication. The patients must be counseled for the occurrence of adverse drug reactions to tinidazole and be made aware to report them, so that the morbidity and mortality associated with the use of the drug can be reduced.

\section{Acknowledgements}

None.

\section{Conflict of interest}

The author declares no conflict of interest.

\section{References}

1. Noriyuki N, Deborah M, John H, et al. Current treatment options for Dientamoeba fragilis infections. International Journal for Parasitology:Drugs and Drug Resistance. 2012;2:204-215.

2. Singbal SS, Rataboli PV. Anaphylaxis and hypersensitivity syndrome reactions in increasing severity following repeated exposure to tinidazole. J Postgrad Med. 2005;51(3):243-244.

3. Sangma K, Wahlang J, Marak M, et al. Fixed drug eruption due to tinidazole: 2 case reports and review of literature. The Internet Journal of Pharmacology. 2012;10(1):174-179.

4. Arika B, Rashmi K, Ramam M. Fixed drug eruption due to cross reaction between two azoles used for different indications. Indian J Dermatol Venereol Leprol. 2008;74(1):81.

5. Jose VM, Sarafudheen V. An unusual adverse effect of tinidazole. Indian Journal of Pharmacology. 2002;34:434-435.

6. Jafferany M, Haroon TS. Tinidazole - induced fixed drug eruption. $J$ Pak Med Assoc. 1987;37(5):136-137.

7. Aase S, Olsen AK, Roland M, et al. Severe Toxic Reaction to Tinidazole. Eur J clin pharmacol. 1983;24(3):425-427.

8. Chandler S, Gautam LS. Fixed dose drug combinations (FDCs): rational or irrational: a view point. Br J Clin Pharmacol. 2008;65(5):795-796. 